\title{
Complete genome sequence of the phenanthrene-degrading soil bacterium Delftia acidovorans Cs1-4
}

Ameesha R. Shetty ${ }^{1}$, Vidya de Gannes ${ }^{2}$, Chioma C. Obi ${ }^{3}$, Susan Lucas ${ }^{4}$, Alla Lapidus ${ }^{5}$, Jan-Fang Cheng ${ }^{4}$, Lynne A. Goodwin' ${ }^{6}$, Samuel Pitluck ${ }^{4}$, Linda Peters ${ }^{4}$, Natalia Mikhailova ${ }^{4}$, Hazuki Teshima ${ }^{6}$, Cliff Han ${ }^{6}$, Roxanne Tapia ${ }^{6}$, Miriam Land ${ }^{7}$, Loren J. Hauser ${ }^{7}$, Nikos Kyrpides ${ }^{4}$, Natalia Ivanova ${ }^{4}$, Ioanna Pagani ${ }^{4}$, Patrick S. G. Chain ${ }^{6}$, Vincent J Denef ${ }^{8}$, Tanya Woyke ${ }^{4}$ and William J. Hickey ${ }^{1 *}$

\begin{abstract}
Polycyclic aromatic hydrocarbons (PAH) are ubiquitous environmental pollutants and microbial biodegradation is an important means of remediation of PAH-contaminated soil. Delftia acidovorans Cs1-4 (formerly Delftia sp. Cs1-4) was isolated by using phenanthrene as the sole carbon source from PAH contaminated soil in Wisconsin. Its full genome sequence was determined to gain insights into a mechanisms underlying biodegradation of PAH. Three genomic libraries were constructed and sequenced: an Illumina GAii shotgun library (916,416,493 reads), a 454 Titanium standard library (770,171 reads) and one paired-end 454 library (average insert size of 8 kb, 508,092 reads). The initial assembly contained 40 contigs in two scaffolds. The 454 Titanium standard data and the 454 paired end data were assembled together and the consensus sequences were computationally shredded into $2 \mathrm{~kb}$ overlapping shreds. Illumina sequencing data was assembled, and the consensus sequence was computationally shredded into $1.5 \mathrm{~kb}$ overlapping shreds. Gaps between contigs were closed by editing in Consed, by PCR and by Bubble PCR primer walks. A total of 182 additional reactions were needed to close gaps and to raise the quality of the finished sequence. The final assembly is based on $253.3 \mathrm{Mb}$ of 454 draft data (averaging $38.4 \mathrm{X}$ coverage) and $590.2 \mathrm{Mb}$ of Illumina draft data (averaging $89.4 \mathrm{X}$ coverage). The genome of strain Cs1-4 consists of a single circular chromosome of 6,685,842 bp ( $66.7 \% \mathrm{G}+\mathrm{C})$ containing 6,028 predicted genes; 5,931 of these genes were protein-encoding and 4,425 gene products were assigned to a putative function. Genes encoding phenanthrene degradation were localized to a $232 \mathrm{~kb}$ genomic island (termed the phn island), which contained near its 3' end a bacteriophage P4-like integrase, an enzyme often associated with chromosomal integration of mobile genetic elements. Other biodegradation pathways reconstructed from the genome sequence included: benzoate (by the acetyl-CoA pathway), styrene, nicotinic acid (by the maleamate pathway) and the pesticides Dicamba and Fenitrothion. Determination of the complete genome sequence of $D$. acidovorans CS1-4 has provided new insights the microbial mechanisms of PAH biodegradation that may shape the process in the environment.
\end{abstract}

Keywords: Delftia acidovorans Cs1-4, Genome, phn island, Phenanthrene, polycyclic aromatic hydrocarbons, Nanopods

\footnotetext{
* Correspondence: wjhickey@wisc.edu

${ }^{1}$ O.N. Allen Laboratory for Soil Microbiology, Department of Soil Science,

University of Wisconsin-Madison, Madison, WI 53706, USA

Full list of author information is available at the end of the article
} link to the Creative Commons license, and indicate if changes were made. The Creative Commons Public Domain Dedication waiver (http://creativecommons.org/publicdomain/zero/1.0/) applies to the data made available in this article, unless otherwise stated. 


\section{Introduction}

Polycyclic aromatic hydrocarbons (PAH) are ubiquitous environmental pollutants and microbial biodegradation is an important means of remediation of PAH-contaminated soil. Delftia acidovorans Cs1-4 (formerly Delftia sp. Cs1-4) was isolated using phenanthrene as the sole carbon source from PAH contaminated soil in Wisconsin [1] and its genome sequence was determined to gain insights into the mechanisms and pathways of PAH metabolism. D. acidovorans Cs1-4 was also unique as the strain in which the novel extracellular structures called nanopods were discovered [2]. Nanopods are extensions of the cell that consist of a surface layer protein encasing outer membrane vesicles (Fig. 1; [2]). The specific functions of nanopods in D. acidovorans Cs1-4 are unknown. But, some connection to phenanthrene degradation is likely as growth on that compound induces production of these structures [2] and mutants disabled in production of nanopods are impaired in growth on phenanthrene $[2,3]$.

Bacterial degraders of PAH can be grouped based on the amino acid similarities in the large subunit of the ring hydroxylating dioxygenase, which initiates $\mathrm{PAH}$ metabolism. Based on those RHD similarities, several groups of PAH-degrading bacteria have been resolved [4, 5], and D. acidovorans Cs1-4 is the first representative of the group designated as the Phn family to have a full genome sequence determined. A draft genome sequence has also been determined for a second representative of the Phn family, Burkholderia sp. Ch1-1 (GenBank ADNR00000000; [6]).

Bacteria belonging to different RHD groups typically differ in other characteristics relevant to PAH metabolism including the range of $\mathrm{PAH}$ degraded, pathways for PAH metabolism and the structure of gene clusters encoding PAH degradation [7]. Furthermore, lateral transfer of RHD genes of different phylogenetic groups appears to be mediated by different types of mobile genetic elements [8]. The full genome sequence of $D$. acidovorans Cs1-4 can thus provide insights into a variety of aspects of PAH metabolism in general, and phenanthrene degradation in particular.

\section{Organism information \\ Classification and features}

The genus Delftia is a phylogenetically cohesive group within the Comamonadaceae family of the Betaproteobacteria (Table 1, Fig. 2). At the time of writing, permanent draft genome sequences were publically available for four draft or finished genome sequences of $D$. acidovorans (including D. acidovorans Cs1-4). But, of those four genomes, only those of strains Cs1-4 and SPH1 (NC_010002) appear to be bone fide representatives of this species, as $16 \mathrm{~S}$ rRNA sequences of the other two strains, CCUG 247B and CCUG 15835, best match to Delftia tsuruhatensis (Table 2). These species affiliations were supported by a phylogenetic tree of the Comamonadaceae, which resolved three species clusters within Delftia: D. acidovorans, D. tsruhatensis and D. litopenaei (Fig. 2). Strains CCUG 247B and CCUG 15835 clustered with $D$. tsruhatensis instead of $D$. acidovorans. A fourth species, D. lacustris, clustered with $D$. tsruhatensis and thus, in this analysis, did not have strong phylogenetic support as a distinct species (Fig. 2).

Delftia acidovorans Cs1-4 was isolated from soil contaminated by coal tar at the former site of a manufactured gas plant in Chippewa Falls, WI by using an enrichment culture with phenanthrene as the sole added carbon source [1]. Strain Cs1-4 has since been used as the model organism for the study of nanopods [2, 3]. Delftia acidovorans Cs1-4 is deposited in the culture collection of the United States Department of Agriculture, Agricultural Research Service (Peoria, IL) as strain NRRL B-65277.

The phospholipid fatty acid profile of $D$. acidovorans Cs1-4 (cells grown on Tryptic Soy Broth and PLFA prepared by the Bligh and Dyer method, [9]) was 19:0 CYCLO $\omega 8 \mathrm{c}, 2 \%$; 18:1 $\omega 7 \mathrm{c}, 6$ \%; 18:0, 9 \%; 17:0 CYCLO, $23 \%$; 16:1 $\omega 7 c, 11 \%$; 16:0, $36 \%$; sum of minor PLFA, $14 \%$. The major quinone is ubiquinone. The glycosyl composition of lipopolysaccharide was (mole \%): rhamnose, $49.1 \%$, glucose, $38.0 \%$, N-acetylglucosamine, $10.7 \%$ and Nacetylgalactosemine, $2.2 \%$. The LPS was prepared by a Tri reagent method [10] and monosaccaride content determined by liquid chromatography-mass spectrometry. A distinguishing characteristic of $D$. acidovorans is a novel surface layer protein, NpdA [2] and NpdA of D. acidocvorans Cs1-4 was antigenically distinct form that of $D$. acidovorans 15668 and SPH1 (Fig. 3). Immunoblotting was done following the method of Gallager et al. 2001 [11] with anti-NpdA prepared as described by Shetty et al. 2011 [2].

Biolog GN plates (Biolog, Hayward, CA) were used to assay metabolic characteristics of D. acidovorans Cs1-4, as well as D. acidovorans strains SPH1 and ATCC 15668. All three were positive for metabolism of: Tween 80, Tween 40, D-mannitol, D-psicose, pyruvic acid methyl ester, succinic acid mono-methyl-ester, cis-aconitic acid, formic acid, D-gluconic acid, $\alpha$-hydroxybutyric acid, $\beta$-hydroxybutyric, $\gamma$-hydroxybutyric acid, $\beta$-hydroxyphenylacetic acid, itaconic acid, $\alpha$-ketobutyric acid, $\alpha$-ketoglutaric acid, $\alpha$-ketovaleric acid, L-lactic acid, malonic acid, propionic acid, quinic acid, D-saccharic acid, sebacic acid, succinic acid, bromosuccinic acid, L-alaninamide, D-alanine, L-alanine, L-alanylglycine, L-aspartic acid, L-histidine, L-leucine, L-phenylalanine, L-proline, L-pyroglutamic acid, L-threonine, $\gamma$-amino butyric acid, urocanic acid and glycerol.

Strains Cs1-4, SPH1 and ATCC 15668 were negative for metabolism of: $\alpha$-cyclodextrin, dextrin, glycogen, $N$ acetyl-D-galactosamine, $\mathrm{N}$-acetyl-D-glucosamine, adonitol, L-arabinose, D-arabitol, D-cellobiose, L-fructose, 

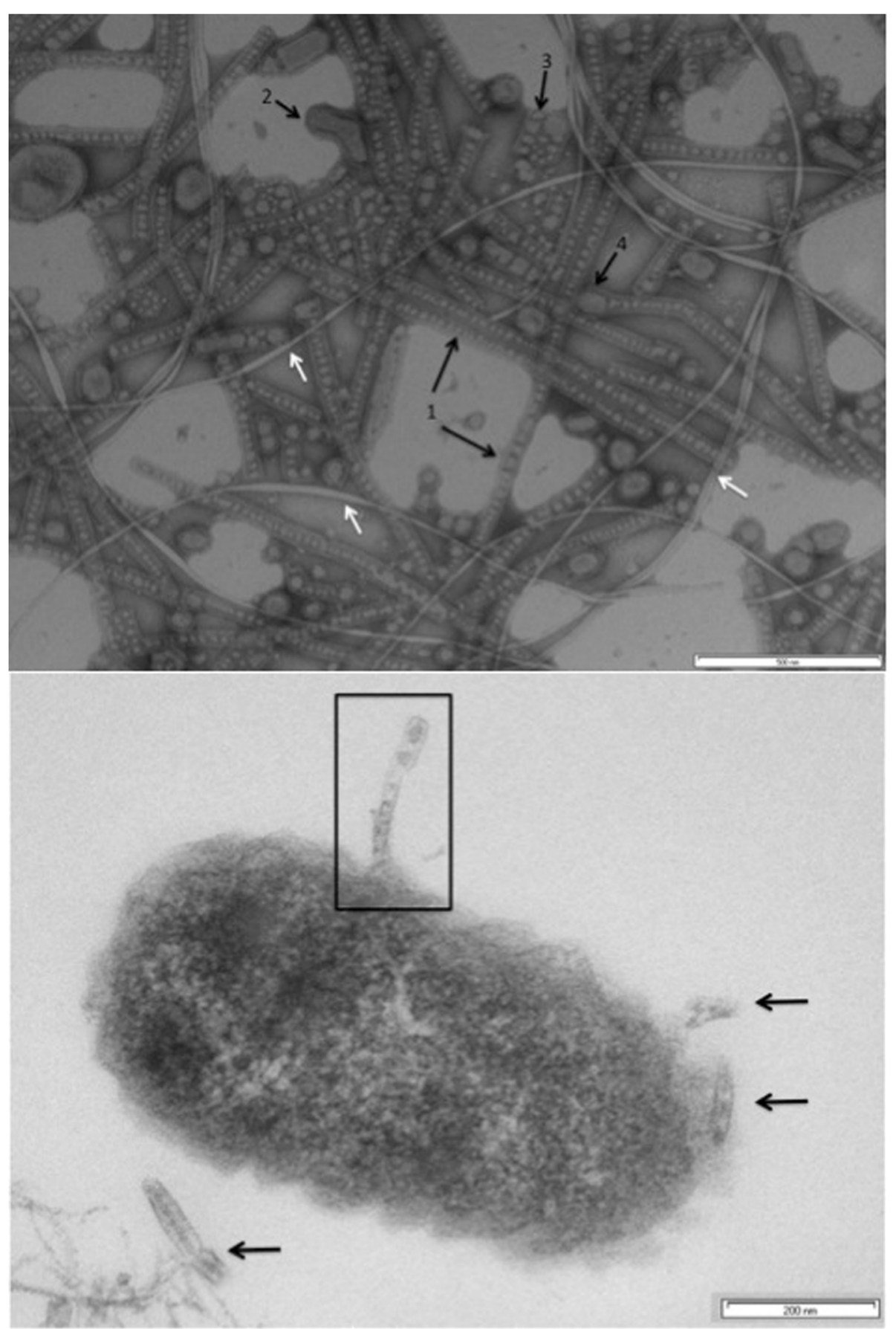

Fig. 1 Top panel: Transmission electron micrograph of negatively-stained culture fluids from phenanthrene-grown D. acidovorans Cs1-4. Nanopods and related structures are indicated by the black arrows, flagella are shown by white arrows. Features indicated by numbers are: 1.) linear structures, 2.) globular elements, 3.) blocky formations and 4.) linear structure with globular element at terminus. The scale bar at bottom right represents $500 \mathrm{~nm}$. Bottom panel: Thin section transmission electron micrograph of D. acidovorans Cs1-4 cell grown as a biofilm on phenanthrene. A nanopod growing from the cell surface is enclosed by the box and arrows indicate segments of other nanopods in the biofilm matrix. The scale bar at bottom right represents $200 \mathrm{~nm}$

$\mathrm{D}$-galactose, gentiobiose, $\alpha$-D-glucose, $\alpha$-D-lactose, lactulose, maltose, $\mathrm{D}$-mannose, $\mathrm{D}$-melibiose, $\beta$-methyl$\mathrm{D}$-glucoside, D-raffinose, L-rhamnose, D-sorbitol, sucrose, D-trehalose, turanose, xylitol, D-galactonic acid lactone, D-galacturonic acid, D-glucosaminic acid, Dglucuronic acid, glycyl-L-aspartic acid, glycyl-L-glutamic acid, hydroxyl-L-proline, L-ornithine, D-serine, L-serine, D-melibiose, L-carnitine, inosine, uridine, thymidine, 
Table 1 Classification and general features of Delftia acidovorans Cs1-4 according to MIGS recommendation [20]

\begin{tabular}{|c|c|c|c|}
\hline MIGS ID & Property & Term & $\begin{array}{l}\text { Evidence } \\
\text { code }^{\mathrm{a}}\end{array}$ \\
\hline & Current & Domain Bacteria & TAS [26] \\
\hline & & Phylum Proteobacteria & TAS [27] \\
\hline & & Class Betaproteobacteria & $\begin{array}{l}\text { TAS [28, } \\
29]\end{array}$ \\
\hline & & Order Burkholderiales & $\begin{array}{l}\text { TAS [30, } \\
29]\end{array}$ \\
\hline & & Family Comamonadaceae & TAS [31] \\
\hline & & Genus Delftia & TAS [32] \\
\hline & & Species acidovorans & TAS [32] \\
\hline & Gram stain & Negative & TAS [2] \\
\hline & Cell shape & Rod & TAS [2] \\
\hline & Motility & Motile & TAS [2] \\
\hline & Sporulation & Nonsporulating & TAS [2] \\
\hline & $\begin{array}{l}\text { Temperature } \\
\text { range }\end{array}$ & Mesophilic & TAS [2] \\
\hline & $\begin{array}{l}\text { Optimum } \\
\text { temperature }\end{array}$ & $30^{\circ} \mathrm{C}$ & TAS [2] \\
\hline & $\begin{array}{l}\text { pH range, } \\
\text { optimum }\end{array}$ & $5.5-7.5,7.0$ & TAS [2] \\
\hline & Carbon source & $\begin{array}{l}\text { Phenanthrene, pyruvate, vanillate, } \\
\text { succinate, Formic acid, gluconic } \\
\text { acid, malonic acid, hydroxybutyric } \\
\text { acid, lactic acid, propionic acid }\end{array}$ & TAS [2] \\
\hline MIGS-6 & Habitat & Oil fields, sludge, freshwater, soil & TAS [2] \\
\hline MIGS-6.3 & Salinity & Fresh water only & TAS [2] \\
\hline MIGS-22 & $\begin{array}{l}\text { Oxygen } \\
\text { requirement }\end{array}$ & Aerobic & TAS [2] \\
\hline MIGS-15 & $\begin{array}{l}\text { Biotic } \\
\text { relationship }\end{array}$ & Free-living & TAS [2] \\
\hline MIGS-14 & Pathogenicity & None & TAS [2] \\
\hline MIGS-4 & $\begin{array}{l}\text { Geographic } \\
\text { location }\end{array}$ & Chippewa Falls, Wisconsin, USA & TAS [2] \\
\hline MIGS-5 & $\begin{array}{l}\text { Sample } \\
\text { collection }\end{array}$ & 2003 & TAS [2] \\
\hline MIGS-4.1 & Latitude & $44.9369^{\circ} \mathrm{N}$ & TAS [1] \\
\hline MIGS-4.2 & Longitude & $91.3928^{\circ} \mathrm{W}$ & TAS [1] \\
\hline MIGS-4.4 & Altitude & $256 \mathrm{~m}$ & TAS [1] \\
\hline
\end{tabular}

a Evidence codes - IDA: Inferred from Direct Assay; TAS: Traceable Author Statement (i.e., a direct report exists in the literature); NAS: Non-traceable Author Statement (i.e., not directly observed for the living, isolated sample, but based on a generally accepted property for the species, or anecdotal evidence). These evidence codes are from the Gene Ontology project [33]

phenyethylamine, putrescine, 2-aminoethanol, 2,3butanediol, L- $\alpha$-glycerol phosphate, $\alpha$-D-glucose1-phosphate and D-glucose-6-phosphate.

Delftia acidovorans strains Cs1-4, SPH1 and ATCC 15668 varied in metabolism of six substrates: $i$-erythritol,
D-fructose, $m$-inositol, citric acid, glucuronamide and Lasparagine. Strain Cs1-4 was positive for only $i$-erythritol, strain ATCC 15688 was positive for D-fructose and citric acid, while strain SPH1 metabolized all six of these compounds.

\section{Genome sequencing and annotation Genome project history}

The genome sequence was completed in May 2011 and presented for public access on December 2011 and is available in GenBank (NC_015563). Quality assurance of the genomic DNA preparation used for sequencing was done in the laboratory of W.J. Hickey at the University of Wisconsin-Madison. Sequencing, finishing and annotation were performed by the U.S. DOE JGI. A summary of the project information is shown in Table 3.

\section{Growth conditions and genomic DNA preparation}

Delftia acidovorans Cs1-4 was grown aerobically in $\mathrm{Nu}$ trient Broth at $30{ }^{\circ} \mathrm{C}$. DNA was isolated from $100 \mathrm{~mL}$ of overnight culture by a CTAB method [12]. Cells were collected by centrifugation $(10,000 \times g 10 \mathrm{~min})$ and then resuspended in Tris-EDTA buffer to OD600 of 1.0. Lysozyme $(100 \mathrm{mg} / \mathrm{mL})$ was added followed by $10 \%$ SDS and proteinase $\mathrm{K}(10 \mathrm{mg} / \mathrm{mL})$. After incubation for $1 \mathrm{~h}$ at $37{ }^{\circ} \mathrm{C}, 5 \mathrm{M} \mathrm{NaCl}$ and $\mathrm{CTAB} / \mathrm{NaCl}$ were added, and the solution incubated at $65{ }^{\circ} \mathrm{C}$ for $10 \mathrm{~min}$. DNA was purified by phenol chloroform extraction, and then re-suspended in TE buffer with RNase $(10 \mathrm{mg} / \mathrm{mL})$. For quality confirmation, the DNA preparation was run on a $1 \%$ agarose gel with phage $\lambda$ DNA as a mass standard (DOE JGI).

\section{Genome sequencing and assembly}

The draft genome of $D$. acidovorans Cs1-4 was generated at the DOE JGI using a combination of Illumina and 454 technologies. An Illumina GAii shotgun library which generated 16,416,493 reads totaling $591 \mathrm{Mb}$, a 454 Titanium standard library which generated 770,171 reads and 1 paired end 454 library with an average insert size of $8 \mathrm{~kb}$ which generated 508,092 reads totaling $372.8 \mathrm{Mb}$ of 454 data, was constructed and sequenced. The initial draft assembly contained 40 contigs in 2 scaffolds. The 454 Titanium standard data and the 454 paired end data were assembled together with Newbler, version 2.3. The Newbler consensus sequences were computationally shredded into $2 \mathrm{~kb}$ overlapping shreds. Illumina sequencing data was assembled with VELVET, version 0.7.63, and the consensus sequence was computationally shredded into $1.5 \mathrm{~kb}$ overlapping shreds. We integrated the 454 Newbler consensus shreds, the Illumina VELVET consensus shreds and the read pairs in the 454 paired end library using parallel phrap, version SPS - 4.24 (High Performance Software, LLC). The 


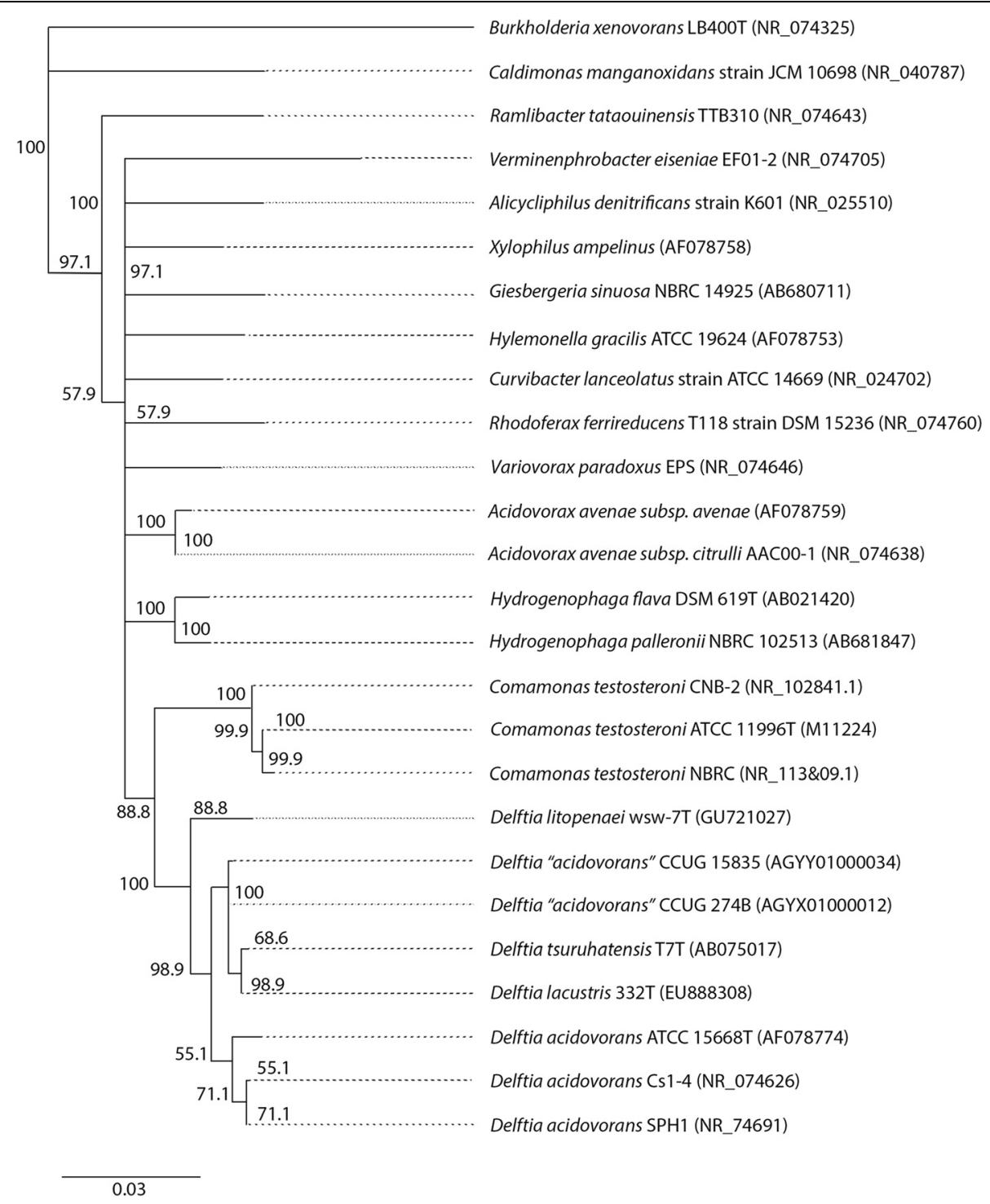

Fig. 2 Phylogenetic tree highlighting the position of Delftia acidovorans Cs1-4 relative to other Delftia species as well as genera within the family Comamonadaceae. The $16 \mathrm{~S}$ rRNA gene sequences were obtained from either type strains (strain designation ends with "T") and/or have full genome sequence data that is publicly available (GenBank accession numbers are indicated in parentheses) were aligned by using MUSCLE (http:// www.drive5.com/muscle/). MEGA v. 5.05 was used for phylogenetic reconstruction via the maximum-likelihood method (tree inference by nearest neighbor interchange), with the Tamura-Nei substitution model. Uniform bootstrap values obtained from 1000 replicate analyses are indicated at nodes. Burkholderia xenovorans LB400 (family Burkholderiaceae) was used as the outgroup. The scale bar represents a $0.03 \%$ nucleotide sequence divergence

Table 2 Summary of currently available genome sequence data from bacteria identified as Delftia acidovorans

\begin{tabular}{|c|c|c|c|c|c|c|c|}
\hline Genome Name & Taxon OID & Status & $\begin{array}{l}\text { 16S rRNA } \\
\text { Gene ID }\end{array}$ & $\begin{array}{l}\text { 16S rRNA } \\
\text { Size(bp) }\end{array}$ & BLAST-N Match & Identity & $\begin{array}{l}\text { Accession Number of } \\
\text { BLAST-N Match }\end{array}$ \\
\hline Delftia acidovorans Cs1-4 & 650716032 & Finished & 650858110 & 1521 & Delftia acidovorans Cs1-4 & $100 \%$ & NR_074626 \\
\hline Delftia acidovorans SPH-1 & 641228489 & Finished & 641295730 & 1526 & Delftia acidovorans SPH-1 & $100 \%$ & NR_074691 \\
\hline Delftia acidovorans CCUG 15835 & 2541046983 & Draft & 2541343813 & 1521 & Delftia tsuruhatensisARB-1 & $100 \%$ & KC572558 \\
\hline Delftia acidovorans CCUG 274B & 2541046984 & Draft & 2541347625 & 1521 & Delftia tsuruhatensisARB-1 & $100 \%$ & KC572558 \\
\hline
\end{tabular}

${ }^{\mathrm{a}}$ Gene ID is as assigned by the JGI Integrated Microbial Genomes database

${ }^{\mathrm{b}}$ Genome sequence determined under the name Delftia sp. Cs1-4 


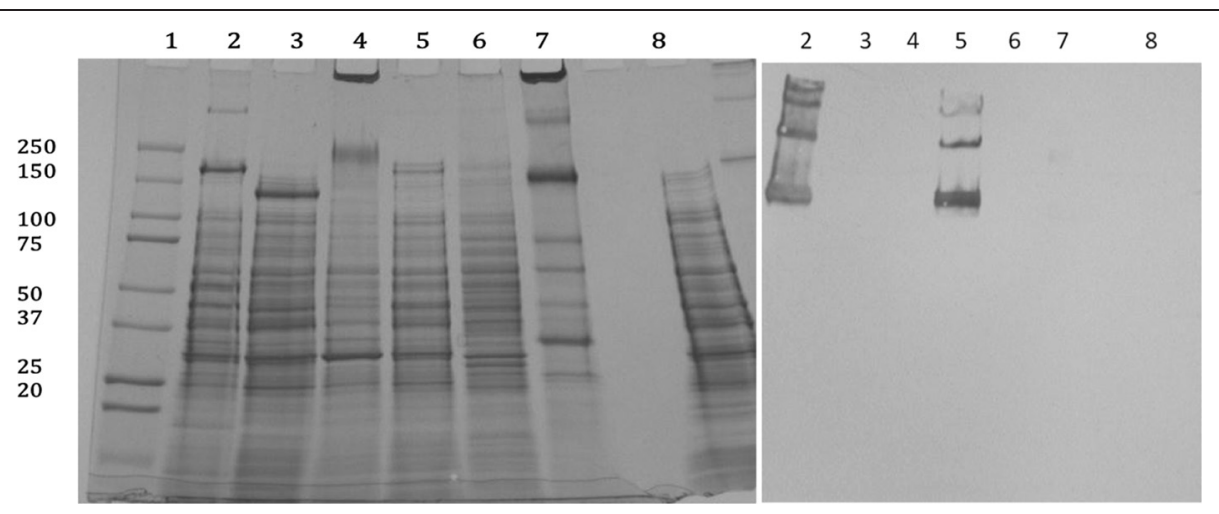

Fig. 3 Antigenic relation of NpdA from Delftia acidovorans CS1-4 with other members of Comamonadaceae family. (Left panel) SDS PAGE . (right panel) Immunoblot with anti-NpdA antibody. Lane 1, Molecular weight marker; lane 2, Delftia acidovorans Cs1-4 (phenanthrene-grown); lane 3, Delftia acidovorans SPH1; lane 4, Deftia acidovorans ATCC15688; lane 5, Delftia acidovorans Cs1-4 (nutrient broth-grown); lane 6, Acidovorax delafieldii 2AN; lane 7, Verminephrobacter eisenae EF01-2; Lane 8, M3. Each lane was loaded with 50 mg of protein, and the gel stained with Coomasie blue. Preparation of anti-NpdA IgY and immunoblotting procedures are described in Shetty et al. [2]

software Consed was used in the following finishing process. Illumina data was used to correct potential base errors and increase consensus quality using the software Polisher developed at JGI (Alla Lapidus, unpublished). Possible mis-assemblies were corrected using gapResolution (Cliff Han, unpublished), Dupfinisher, or sequencing cloned bridging PCR fragments with subcloning. Gaps between contigs were closed by editing in Consed, by PCR and by Bubble PCR primer walks (J-F Cheng, unpublished). A total of 182 additional reactions were necessary to close gaps and to raise the quality of the finished sequence. The total size of the genome is $6,685,842 \mathrm{bp}$ and the final assembly is based on $253.3 \mathrm{Mb}$ of 454 draft data which provides an average $38.4 \times$ coverage of the genome and $590.2 \mathrm{Mb}$ of Illumina draft data which provides an average $89.4 \times$ coverage of the genome.

Table 3 Project information

\begin{tabular}{lll}
\hline MIGS ID & Property & Term \\
\hline MIGS-31 & Finishing quality & Level 6 Finished \\
MIGS-28 & Libraries used & $\begin{array}{l}\text { Titanium draft library, paired end } \\
\text { library and Illumina library }\end{array}$ \\
MIGS-29 & Sequencing platforms & 454 Titanium, Illumina \\
MIGS-31.2 & Fold coverage & $\begin{array}{l}38.4 \times 454 \text { Titanium, 89.4× } \\
\text { Illumina }\end{array}$ \\
MIGS-30 & Assemblers & Newbler, Velvet \\
MIGS-32 & Gene calling method & Prodigal, GenePRIMP \\
& Locus tag & DelCs14 \\
& Genbank ID & NC_015563 \\
& Genbank Date of Release & December 1, 2011 \\
& GOLD ID Bioproject & Gc0178467319 67319 \\
MIGS-13 & Source Material Identifier & Cs1-4 \\
& Project relevance & Biotechnological, Environmental \\
\hline
\end{tabular}

\section{Genome annotation}

Genes were identified using Prodigal as part of the Oak Ridge National Laboratory genome annotation pipeline, followed by a round of manual curation using the JGI GenePRIMP pipeline. The predicted CDSs were translated and used to search the National Center for Biotechnology Information non-redundant database, UniProt, TIGRFam, Pfam, PRIAM, KEGG, COG, and InterPro databases. These data sources were combined to assert a product description for each predicted protein. Non-coding genes and miscellaneous features were predicted using tRNAscan-SE, RNAMMer, Rfam, TMHMM, and signalP. The final genome sequence is deposited in GenBank under accession NC_015563.

\section{Genome properties}

The genome of strain Cs1-4 consists of a single circular chromosome of 6,685,842 bp (66.7 \% G + C content) containing with 6,028 predicted genes (Fig. 4, Table 4). There were 5,931 protein-encoding genes; 4,425 of these gene products were assigned to a putative function with the remaining annotated as hypothetical proteins (Table 5). There were 80 tRNA genes and five 16S rRNA genes. For the latter, three were $100 \%$ identical to each other, while two were $99 \%$ identical (DelCs14_R0076, DelCs14_R0098).

\section{Insights from the genome sequence Comparisons with other sequenced Delftia genomes}

The genome of strain Cs1-4 was similar to that of the other available Delftia genomes with respect to categories such as gene count and \% G + C (Table 4). However, there was a clear distinction between these strains in the area of ribosomal genes, as their abundance was nearly identical in the genomes of strains Cs1-4 and SPH1, but much greater than that in 


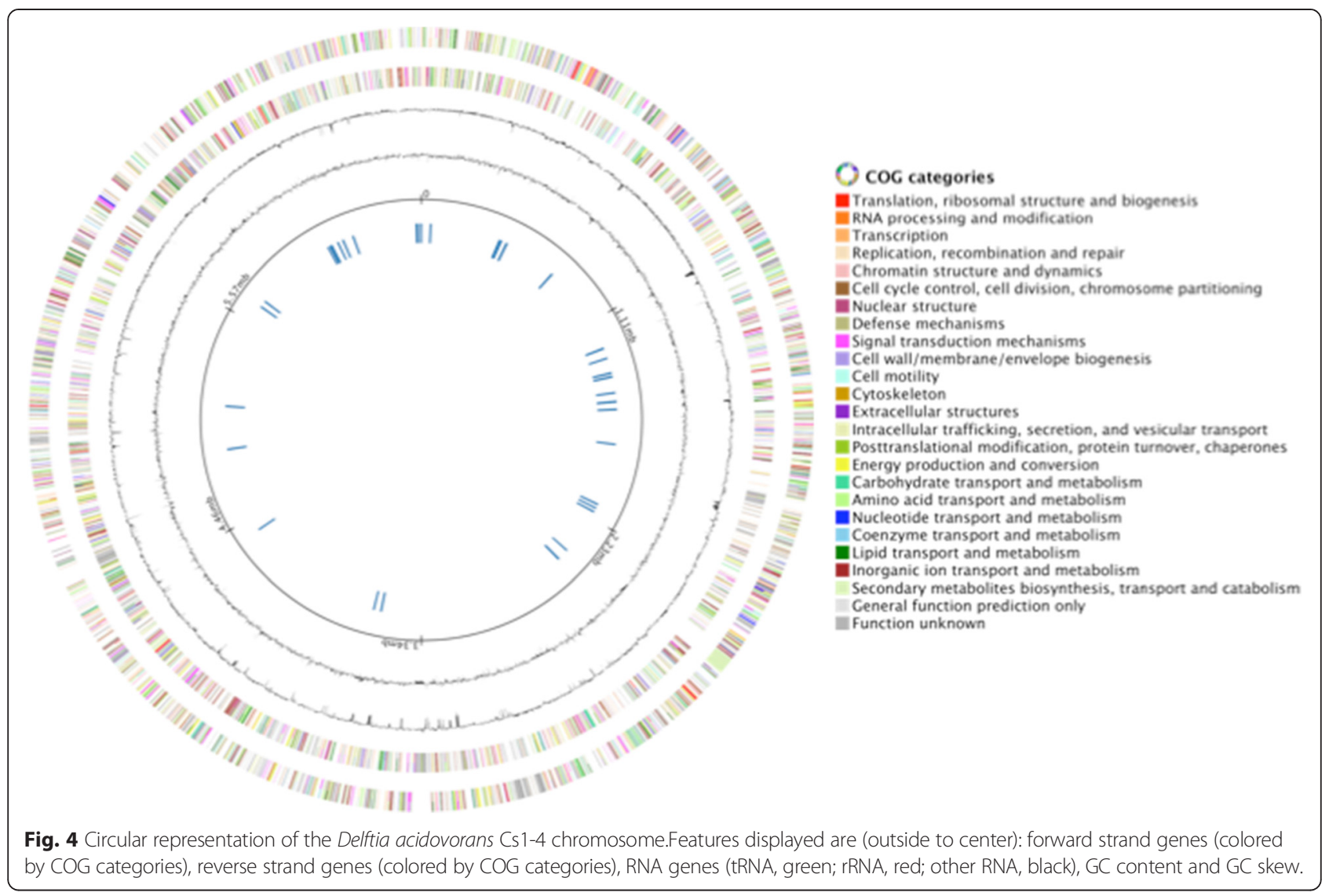

Table 4 Delftia acidovorans Cs1-4 genome statistics

\begin{tabular}{lrc}
\hline Attribute & \multicolumn{1}{l}{ Value } & \% of Total \\
\hline Genome size (bp) & $6,685,842$ & 100 \\
DNA coding (bp) & $5,998,883$ & 89.73 \\
DNA G + C content (bp) & $4,460,657$ & 66.72 \\
DNA scaffold & 1 & 1.00 \\
Total genes & 6,028 & 100.00 \\
Protein coding genes & 5,931 & 98.39 \\
RNA genes & 97 & 16.1 \\
Pseudo genes & 70 & 1.16 \\
Genes in internal clusters & 857 & 14.22 \\
Genes with function prediction & 4,425 & 73.41 \\
Genes assigned to COGs & 4,845 & 80.37 \\
Genes assigned to Pfam domains & 5,061 & 83.96 \\
Genes with signal peptides & 2,505 & 41.56 \\
Genes with transmembrane helices & 1,377 & 22.84 \\
CRISPR repeats & 3 & NA \\
\hline
\end{tabular}

strains CCUG 274B and CCUG 15835. There was also a marked difference between the organisms in the area of function prediction, with strains Cs1-4 and SPH1 having a much larger portion of genes with function predicted than did strains CCUG 274B and CCUG 15835. This divergence of the latter two strains from strains Cs1-4 and SPH1 would further support re-assignment of strains CCUG 274B and CCUG 15835 to a Delftia species other than acidovorans (e.g., D. tsruhatensis).

The closest relative of $D$. acidovorans Cs1-4 with genome sequence data is D. acidovorans SPH1. Strain SPH1 was isolated from a sewage treatment plant in Germany as a part of a microbial consortium that degraded linear alkylbenzene sulfonates [13]. Strains Cs1-4 and SPH1 had $99 \%$ identity over the full length of the 16S rRNA gene. Compared to the type strain $D$. acidovorans ATCC 15668, both strains Cs1-4 and SPH1 had $99 \%$ identity over $99 \%$ of the $16 \mathrm{~S}$ rRNA gene. The genomes of strain Cs1-4 and strain SPH1 had an average nucleotide identity (ANI) of $97.48 \%$, which qualified the strains as the same species using the $95 \%$ ANI threshold [14, 15]. Based on this high level of nucleotide sequence similarity, a common species is reasonable for these bacteria. But, while the genomic composition of strains SPH1 and 
Table 5 Number of genes in Delftia acidovorans Cs1-4 associated with the 25 general COG functional categories

\begin{tabular}{lrrl}
\hline Code & Value & \% of total & Description \\
A & 207 & 3.78 & Translation \\
K & 2 & 0.04 & RNA processing and modification \\
L & 581 & 10.62 & Transcription \\
B & 168 & 3.07 & Replication, recombination and repair \\
D & 2 & 0.04 & Chromatin structure and dynamics \\
V & 32 & 0.58 & Cell cycle control, mitosis and meiosis \\
T & 71 & 1.3 & Defense mechanisms \\
M & 362 & 6.62 & Signal transduction mechanisms \\
N & 251 & 4.59 & Cell wall/membrane biogenesis \\
U & 138 & 2.52 & Cell motility \\
O & 145 & 2.65 & Intracellular trafficking and secretion \\
& 162 & 2.96 & Posttranslational modification, protein \\
C & 346 & 6.32 & Energy production and conversion \\
G & 242 & 4.42 & Carbohydrate transport and metabolism \\
E & 479 & 8.76 & Amino acid transport and metabolism \\
F & 88 & 1.61 & Nucleotide transport and metabolism \\
H & 191 & 3.49 & Coenzyme transport and metabolism \\
I & 261 & 4.77 & Lipid transport and metabolism \\
P & 368 & 6.73 & Inorganic ion transport and metabolism \\
Q & 172 & 3.14 & Secondary metabolites biosynthesis, \\
& & & transport and catabolism \\
R & 621 & 11.35 & General function prediction only \\
S & 580 & 10.60 & Function unknown \\
- & 1183 & 19.63 & Not in CoGs \\
\hline The tota is barnover, chaperones \\
\hline
\end{tabular}

The total is based on the total number of protein coding genes in the annotated genome

Cs1-4 was similar, dot plots exhibited an " $\mathrm{X}$ " alignment (Fig. 5), which is indicative of inversions about the origin of replication [16].

Alignment of the D. acidovorans Cs1-4 and SPH1 genomes revealed in the former a large genomic island $(232 \mathrm{~kb})$ termed the phn island that was absent from strain SPH1 [6]. The island contained near its 3' end a bacteriophage P4-like integrase, a type of enzyme often associated with chromosomal integration of mobile genetic elements [17]. Numerous close orthologs of the strain Cs1-4 integrase can be identified by BLAST-P searches of Genbank, and these may serve as starting points for the elucidation of mobile genetic elements possibly related to the $p h n$ island.

Conversely, genomic alignment of strains Cs1-4 and SPH1 revealed in strain SPH1 a mobile genetic element of $c a .67 \mathrm{~kb}$, which was absent from strain Cs1-4. The loci bounding this element in strain SPH1 were (gene name, equivalent loci in strain Cs1-4) Daci_1694 (rpoH,

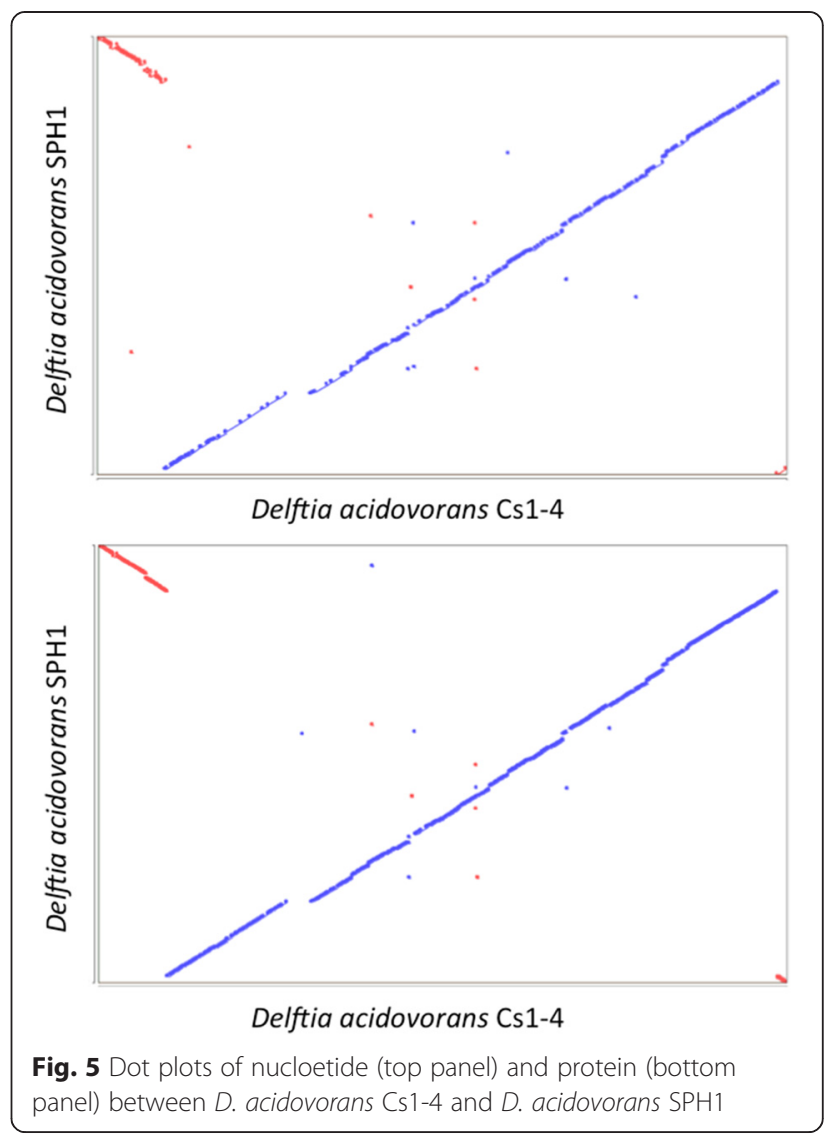

Delcs14_4885) and Daci_1756 (inclR, Delcs14_4884). The mobile genetic element in strain SPH1 contained metal detoxification functions, and had extensive similarity to a region in the genomes of strains CCUG 274B and CCUG 15835.

The secretory machinery of strain Cs1-4 were similar to strain SPH1, as well as to strains CCUG 274B and CCUG 15835. These consisted of Type II, Type IV and Type VI secretion system (T6SS) along with the components of a Sec-Signal Recognition Particle Translocon and the tat $A B C$ genes of the Twin-Arginine Translocation pathway. For all strains, there was a single T6SS cluster. The functions of T6SS have been explored mostly in pathogenic bacteria, where a common feature is mediation of intercellular contact in antagonistic interactions [18]. Functions of T6SS in environmental bacteria such as the $D$. acidovorans strains discussed here are unknown.

Delftia acidovorans Cs1-4 produces a novel surface layer protein, which is essential for the formation of extracellular structure called nanopods [2]. The surface layer protein (termed Nanopod Protein A, NpdA) is encoded by DelCs14_5206 (npdA), and orthologs of $n p d A$ are present in the genomes of $D$. acidovorans SPH1 as well as D. acidovorans strains CCUG 274B and CCUG 15835. 


\section{Profiles of metabolic networks and pathways} Characterization of phenanthrene catabolism genes

Genes for the entire phenanthrene catabolic pathway were identified on a novel $232 \mathrm{~kb}$ genomic island named the phn island [6] and were segregated into three clusters that were predicted to encode the metabolism of phenanthrene to ortho-phthalate (phn genes), ortho-phthalate to protocatechuate (oph genes) and meta-cleavage of protocatechuate to pyruvate and oxaloacetate (pmd genes). These clusters were non-contiguous; the oph and pmd clusters were separated from each other by $c a .5 \mathrm{~kb}$ and located $60 \mathrm{~kb}$ upstream of the phn cluster. The phn gene cluster had a $\% \mathrm{G}+\mathrm{C}$ content of $c a$. 50, which differed significantly from the $66.7 \% \mathrm{G}+\mathrm{C}$ content of the Cs1-4 chromosome, while $\% \mathrm{G}+\mathrm{C}$ of the $o p h$ and pmd genes was similar to the chromosomal backbone. The $\mathrm{G}+\mathrm{C}$ content of Comamonadacea genomes ranges from $60 \%$ to $70 \%$, thus the phylogenetic origin of the phn genes is outside of this family and likely outside of the order Burkholderiales.

\section{Styrene degradation via the phenyl acetate pathway}

Styrene is often a soil pollutant and strain Cs1-4 possessed genes predicted to encode its degradation by the phenylacetate pathway. The putative styrene operon (conversion of styrene to phenyl acetate [19]), consisted of a regulatory element (marR, DelCs14_4846), dienelactone hydrolase (DelCs14_4847), flavin reductase (styB, DelCs14_4848), monooxygense (styA, DelCs14_48449), short chain dehydrogenase, (DelCs14_4850) and AraCtype transcriptional regulator (DelCs14_4851).

The genetics of phenyl acetate metabolism has been studied in a variety of bacteria, and thirteen genes encoding its transformation to succinyl-CoA and acetyl-CoA have been identified $[20,21]$. These genes often occur in a single cluster, but in strain Cs1-4, they are dispersed across the genome. The single largest cluster contained paaABCDE (DelCs14_5720-24) and paaK (DelCs14_5725), which were predicted to encode a phenylacetyl-CoA epoxidase and phenylacetate-CoA ligase, respectively. Other orthologs that were identified included an epoxyphenylacetyl-CoA isomerase (paaG, DelCs14_0512) and a ring-opening enzyme (paaN, DelCs14_5726).

\section{Benzoate degradation by the benzoyl-CoA pathway}

Metabolism of benzoate is important in the degradation of many aromatic compounds, and benzoate degradation by aerobic bacteria is most commonly initiated by oxygenases. In contrast, growth of strain Cs1-4 on benzoate was predicted to proceed by an alternative pathway in which benzoyl-CoA is formed as the primary metabolite [22]. The gene cluster putatively conferring this function included an ABC-type transporter (DelCs14_0078-82), a benzoate-CoA ligase (DelCs14_0073) and a benzoate oxygenase (boxABC, DelCs14_0073-0075).

\section{Nicotinic acid metabolism by the maleamate pathway}

Biodegradation of nicotinic acid has been widely studied as a model for metabolism of $N$-heterocycles. Also, there is interest in the application of bioremediation of nicotinecontaminated soils at tobacco processing facilities. Jime'nez et al. 2008 [23] conducted a detailed analysis of genes in Pseudomonas putida KT2440 encoding nicotinic degradation by the maleamate pathway, and strain Cs1-4 possesed a similar set of genes. The nic operon in strain Cs1-4 included a Nic regulator (marR, DelCs14_4781), an MFS transporter (nicT, DelCs14_4782), nicotinate dehydrogenase subunit B (nicB2, DelCs14_4783), nicotinate dehydrogenase subunit A (nicA, DelCs14_47840), salicylate 1-monooxygenase (nicC, DelCs14_4785), maleate isomerase (nicE, DelCs14_4786), $N$-formylmaleamate deformylase (nicD, DelCs14_4787), 2,5-dihydroxypyridine 5,6-dioxygenase (nicX, DelCs14_4788), maleamate amidohydrolase (nicF, DelCs14_4789) and an aldehyde dehydrogenase (nicB1, DelCs14_4790).

\section{Pesticide degradation: Dicamba and Fenitrothion}

Two gene clusters in the genome of strain Cs1-4 resembled clusters identified in other proteobacteria that coded for the metabolism of Dicamba (2-methoxy-3,6-dichlorobenzoate) or Fenitrothion (O,O-dimethyl O-[3-methyl-4nitrophenyl] phosphorothioate). The Dicamba cluster in strain Cs1-4 encompassed a 0 -demethylase that was composed of an oxygenase (DelCs14_5158), ferredoxin (DelCs14_5157) and reductase (DelCs14_5158), and the predicted product of this cluster would be 3,6-dichlorosalicylate [24]. The pathway for 3,6-dichlorosalicylate metabolism is not well delineated, but strain Cs1-4 has putative salicylate oxygenases that could mediate ring hydroxylation (e.g., products of DelCs14_3111, DelCs14_3828).

For fenitrothion, biodegradation could occur via methyl hydroquinone as an intermediate, as it possessed a cluster of genes with similarity to those encoding fenitrothion metabolism in Burkholderia sp. NF100 [25]. These genes putatively encoded were a flavoprotein monooxygenase ( $m h q A$, DelCs14_5563), extradiol dioxygenase ( $m$ hqB, DelCs14_5562), a ring hydroxylating oxygenase (DelCs14_5559), ferredoxin (DelCs14_5558) and hydrolase (mhqA, DelCs14_5554).

\section{Conclusions}

Determination of the complete genome sequence of $D$. acidovorans Cs1-4 achieved the objective of enabling new insights into the genes underlying PAH metabolism and evolutionary mechanisms that may shape the process in the environment. Furthermore, the genome sequence data suggested that biodegradative capacity of D. acidovorans Cs1-4 extended beyond PAH, and the organism was well equipped for growth in soils contaminated by a variety of compounds such as $N$-heterocycles and pesticides. 


\section{Abbreviations}

PAH: Polycyclic aromatic hydrocarbons; RHD: Ring hydroxylating dioxygenase; ANI: Average nucleotide identity; NpdA: Nanopod protein A; DOE JGI: Department of Energy Joint Genome Institute; T6SS: Type VI secretion system; LPS: Lipopolysaccaride; PLFA: Phospholipid fatty acid.

\section{Competing interests}

The authors declare that they have no competing interests.

\section{Authors' contributions}

Authors affiliated with the US-DOE were involved in aspects of genome production including sequencing ( $S L, J-F C$, TW), finishing ( $A L, L A G, H T, C H$, RT, PSGC), annotation (ML, LJH, NK, NI, IP, NM) and Genbank submission $(S P, L P)$. All other authors were involved in development of the initial proposal for sequencing of the D. acidovorans Cs1-4 genome (ARS, PSGC, VJD, WJH) and/or manuscript preparation (ARS, VG, CCO, WJH). All authors read and approved the final manuscript.

\section{Acknowledgements}

Sequencing, assembly and computational annotation of the D. acidovorans Cs1-4 genome was done by U.S Department of Energy, Joint Genome Institute through the Community Sequencing Project (CSP795673 to William J. Hickey). The work conducted by the U.S. Department of Energy, Joint Genome Institute was supported by U.S. Department of Energy, Office of Science under contract No. DE-AC02-05CH11231. Research in the corresponding author's laboratory on genomic and phenotypic characteristics of D. acidovorans Cs1-4 was supported by the O.N. Allen Professorship in Soil Microbiology (to William J. Hickey).

\section{Author details}

'O.N. Allen Laboratory for Soil Microbiology, Department of Soil Science, University of Wisconsin-Madison, Madison, WI 53706, USA. ${ }^{2}$ Department of Food Production, University of the West Indies, St. Augustine, Trinidad and Tobago. ${ }^{3}$ Department of Microbiology, University of Lagos, Lagos, Nigeria. ${ }^{4}$ DOE Joint Genome Institute, Walnut Creek, CA, USA. ${ }^{5}$ Algorithmic Biology Lab, St. Petersburg Academic University, St.Petersburg, Russia. ${ }^{6}$ Bioscience Division, Los Alamos National Laboratory, Los Alamos, NM, USA. ${ }^{7}$ Oak Ridge National Laboratory, Oak Ridge, TN, USA. ${ }^{8}$ Department of Ecology and Evolutionary Biology, University of Michigan, Ann Arbor, MI, USA.

Received: 15 July 2014 Accepted: 15 July 2015

Published online: 15 August 2015

\section{References}

1. Vacca DJ, Bleam WF, Hickey WJ. Isolation of soil bacteria adapted to degrade humic acid-sorbed phenanthrene. Appl Environ Microbiol. 2005:71:3797-805.

2. Shetty A, Chen S, Tocheva El, Grant J, Hickey WJ. Nanopods: a new bacterial structure and mechanism for deployment of outer membrane vesicles. PLoS ONE. 2011;6, e20725

3. Shetty A, Hickey WJ. Effects of outer membrane vesicle formation, surfacelayer production and nanopod development on the metabolism of phenanthrene by Delftia acidovorans Cs1-4. PLoS ONE. 2014;9, e92143.

4. Stolz A. Molecular characteristics of xenobiotic-degrading sphingomonads. Appl Microbiol Biotechnol. 2009;81:793-811.

5. Moser R, Stahl U. Insights into the genetic diversity of initial dioxygenases from PAH-degrading bacteria. Appl Microbiol Biotechnol. 2001;55:609-18.

6. Hickey WJ, Chen S, Zhao J. The phn island: a new genomic island encoding catabolism of polynuclear aromatic hydrocarbons. Front Microbiol. 2012;3:1-15.

7. Habe H, Omori T. Genetics of polycyclic aromatic hydrocarbon metabolism in diverse aerobic bacteria. Biosci Biotechnol Biochem. 2003;67:225-43.

8. Top EM, Springael D. The role of mobile genetic elements in bacterial adaptation to xenobiotic organic compounds. Curr Opin Biotechnol. 2003;14:262-9.

9. Bligh EG, Dyer WJ. A rapid method of total lipid extraction and purification. Can J Biochem Phys. 1959;37:911-7.

10. Yi EC, Hackett M. Rapid isolation method for lipopolysaccharide and lipid A from Gram-negative bacteria. Analyst. 2000;125:651-6.
11. Gallagher S, Winston SE, Fuller SA, Hurrell JGR. Immunoblotting and Immunodetection. In: Ausubel R, Bent RE, editors. Protocols in Molecular Biology. New York: John Wiley \& Sons; 2008. p. 10.8.1-10.8.28.

12. Wilson K. Preparation of genomic DNA from bacteria. In: Ausubel R, Bent RE, editors. Current Protocols in Molecular Biology. New York: John Wiley \& Sons: 2001. p. 2.4.1-5.

13. Schleheck D, Knepper TP, Fischer K, Cook AM. Mineralization of individual congeners of linear alkylbenzenesulfonate by defined pairs of heterotrophic bacteria. Appl Environ Microbiol. 2004;70:4053-63.

14. Konstantinidis KT, Ramette A, Tiedje JM. The bacterial species definition in the genomic era. Phil Trans R Soc B. 2006;361:1929-40.

15. Kim M, Oh HS, Park SC, Chun J. Towards a taxonomic coherence between average nucleotide identity and 16S rRNA gene sequence similarity for species demarcation of prokaryotes. Int J Syst Evol Micrbiol. 2014;64:364-51.

16. Eisen J, Heidelberg J, White O, Salzberg S. Evidence for symmetric chromosomal inversions around the replication origin in bacteria. Genome Biol. 2000;1:1-9.

17. Ravatan R, Studer S, Zehnder AJ, van der Meer JR. Int-B13, an unusual site-specific recombinase of the bacteriophage P4 integrase family, is responsible for chromosomal insertion of the 105-kilobase clc element of Pseudomonas sp. strain B13. J Bacteriol. 1998;180:5505-14.

18. Basler M, Ho BT, Mekalanos JJ. Tit-for-tat: Type VI secretion system counterattack during bacterial cell-cell interactions. Cell. 2013;154:884-94

19. Tischler D, Gröning JAD, Kaschabek SR, Schlömann M. One-component styrene monooxygenases: an evolutionary view on a rare class of flavoproteins. Appl Biochem Biotechnol. 2012:167:931-44.

20. Teufel R, Mascaraque V, Ismail W, Voss M, Perera J, Eisenreich W, et al. Bacterial phenylalanine and phenylacetate catabolic pathway revealed. Proc Natl Acad Sci U S A. 2010;107:14390-5.

21. Luengo JM, Garcõ JL, Olivera ER. The phenylacetyl-CoA catabolon: a complex catabolic unit with broad biotechnological applications. Mol Microbiol. 2001;39:1434-42.

22. Harwood CS, Burchhardt G, Herrmann H, Fuchs G. Anaerobic metabolism of aromatic compounds via the benzoyl-CoA pathway. FEMS Microbiol Rev. 1998;22:439-58.

23. Jiménez Jl, Canales A, Jiménez-Barbero J, Ginalski K, Leszek R, García JL, et al. Deciphering the genetic determinants for aerobic nicotinic acid degradation: the nic cluster from Pseudomonas putida KT2440. Proc Natl Acad Sci U S A. 2008:105:11329-34

24. Wang X-Z, Li B, Herman PL, Weeks DP. A three component enzyme system catalyzes the O-demethylation of the herbicide Dicamba in Pseudomonas maltophilia D1-6. Appl Environ Microbiol. 1997;63:1623-6.

25. Tago K, Sato J, Takesa H, Kawagishi H, Hayatsu M. Characterization of methylhydroquinone-metabolizing oxygenase genes encoded on plasmid in Burkholderia sp. NF100. J Biosci Bioeng. 2005;100:517-23.

26. Woese CR, Kandler O, Wheelis ML. Towards a natural system of organisms: proposal for the domains Archaea, Bacteria and Eucarya. Proc Natl Acad Sc U S A. 1990:87:4576-9.

27. Garrity GM, Bell JA, Pylum LT, XIV. Proteobacteria phyl nov. In: Garrity GM, Brenner DJ, Krieg NR, Stanley JT, editors. Bergey's Manual of Systematic Bacteriology, vol. 2. 2nd ed. Springer, New York: Part C; 2005. p. 1.

28. Garrity GM, Bell JA, Lilburn T. Class II. Betaproteobacteria class. nov. In: Garrity GM, Brenner DJ, Krieg NR, Stanley JT, editors. Bergey's Manual of Systematic Bacteriology, Second Edition, Volume 2, Part C. Springer: New York; 2005. p. 575.

29. Validation List No. 107. List of new names and new combinations previously effectively, but not validly, published. Int J Syst Evol Microbiol. 2006:56:1-6.

30. Garrity GM, Bell JA, Lilburn T, Order I. Burkholderiales ord nov. In: Garrity GM, Brenner DJ, Krieg NR, Staley JT, editors. Bergey's Manual of Systematic Bacteriology, vol. 2. 2nd ed. Springer, New York: Part C; 2005. p. 575.

31. Willems A, De Ley J, Gillis M, Kersters K. Comamonadaceae, a new family encompassing the acidovorans rRNA complex, including Variovorax paradoxus gen. nov., comb. nov., for Alcaligenes paradoxus (Davis 1969). Int J Syst Bacteriol. 1991:41:445-50.

32. Wen A, Fegan M, Hayward C, Chakraborty S, Sly LI. Phylogenetic relationships among members of the Comamonadaceae, and description of Delftia acidovorans (den Dooren de Jong 1926 and Tamaoka et al. 1987) gen. nov, comb. nov. Int J Syst Evol Micrbiol. 1999:9:567-76.

33. Ashburner M, Ball CA, Blake JA, Botstein D, Butler H, Cherry JM, et al. Gene Ontology: tool for the unification of biology. The gene ontology consortium. Nat Genet. 2000;25:25-9. 